\title{
A avaliação de impacto à saúde como campo de saber
}

\section{Appraisal of health impacts as knowledge field}

\author{
Carlos Pereira \\ Fundação Oswaldo Cruz. Escola Nacional de Saúde Pública Sérgio \\ Arouca. Rio de Janeiro, RJ, Brasil. \\ E-mail: carlos.rpereiraळhotmail.com

\section{Sandra Hacon} \\ Fundação Oswaldo Cruz. Escola Nacional de Saúde Pública Sérgio \\ Arouca. Rio de Janeiro, RJ, Brasil. \\ E-mail: sandrahaconळgmail.com
}

\section{Correspondência}

Carlos Pereira

Rua Leopoldo Bulhões, I.480, sala 620, Manguinhos. Rio de Janeiro,

RJ, Brasil. CEP 21041-210.

\section{Resumo}

O debate sobre o que é Avaliação de Impacto à Saúde (AIS) e quais são seus domínios tem ocorrido desde os primórdios de sua prática, resultando em uma série de documentos-guia que, apoiados em visões e pressupostos específicos, buscaram elucidar tais questões. Longe de findado esse debate, a atual expansão da utilização da AIS, as adaptações para diferentes contextos e o agregar de conhecimentos e práticas que lhe conferem o status de campo de saber revigoram a necessidade de revisitar conceitos e preceitos sobre tal constructo. Foi essa necessidade que motivou a elaboração deste artigo, que busca resgatar a definição da AIS como um campo de saber de amplo aparato teórico e expor práticas e estudos que poderiam fazer parte do domínio da avaliação de impactos à saúde sob um entendimento mais amplo do que seria esse constructo.

Palavras-chave: Avaliação do Impacto à Saúde; Estudos de Avaliação como Assunto; Impactos na Saúde; Saúde Ambiental. 


\section{Abstract}

The debate on what "appraisal of health impacts" is and which its limits are have remained since the beginning of performing studies practice. In consequence, many documents have been published to elucidate these questions, based on specific premises and approaches, especially for health impacts assessments (HIA). The current expansion of studies, mainly of HIA, the adjustments for different contexts, and the aggregated knowledge which vest it with the status of field of knowledge place the debate far from over, and invigorate the need of revisiting concepts and premises of health impact appraisal constructs. This need has motivated the elaboration of this study, which aims to revive the debate of this construct as a field of knowledge with vast theoretical apparatus, and to discuss, under a wide comprehension, about studies that could belong to this field, besides the HIA ones.

Keywords: Health Impact Assessment; Evaluation Studies as Topic; Impacts on Health; Environmental Health.

\section{Introdução}

As ciências ambientais foram pioneiras na utilização das avaliações de impacto como instrumento de planejamento e gestão. Ao longo do tempo, a necessidade da avaliação de outras vertentes de impacto motivou o surgimento de outras formas e metodologias de análise (Donelly; Dalal-Clayton; Hughes, 1998) que, embora ligadas pelos mesmos fundamentos, buscam embasamento em diferentes teorias (Pope et al., 2013). Um exemplo disso é a Avaliação de Impactos à Saúde (AIS), que se estruturou sob forte influência da avaliação de impacto ambiental e da atuação de movimentos de saúde pública que defendiam a necessidade de melhor abordagem dos efeitos de estratégias de desenvolvimento sobre a saúde humana (HarrisRoxas et al., 2012).

Desde muito cedo, questões como o que é AIS, quais as metodologias aceitas e que habilitações devem ter seus praticantes têm sido debatidas, tanto que em 1999 ocorreu a publicação do Consenso de Gothenburg, no qual foram sugeridos um conceito e uma metodologia, no sentido de padronizar a prática e dirimir as dúvidas concernentes à delimitação da AIS, definida a partir de então, como processo sistemático de estudo e análise.

O Consenso de Gothenburg foi o esforço empreendido pela Organização Mundial de Saúde para estruturação da AIS. Nesse acordo, a AIS é definida como a "combinação de procedimentos, métodos e ferramentas pelos quais políticas, programas e projetos podem ser julgados de acordo com seus efeitos potenciais à saúde de uma população e quanto à distribuição de tais efeitos na população" (WHO, 1999, p. 4, tradução livre) e seus passos metodológicos são basicamente: diagnóstico (screening), definição do escopo (scoping), avaliação (appraisal), elaboração do relatório (reporting), e monitoramento (monitoring and evaluation). Pelo Consenso, o foco é a análise preditiva de impactos com vistas à promoção da saúde e à prevenção de danos. 0 monitoramento, neste caso, é feito para avaliar os desdobramentos do estudo preditivo e a efetividade das ações tomadas.

Sem dúvida, o Consenso foi um marco conceitual no sentido de definir bases metodológicas e 
procedurais para a realização dos estudos chamados de AIS. Como linha de pensamento já organizada, possibilitou o avanço na realização dos estudos, tornando-os compatíveis e comparáveis. Tanto que hoje a maior parte dos estudos elaborados sob o nome de "avaliação de impacto à saúde", independente da língua, segue os procedimentos sugeridos por tal Consenso. Levando em consideração que no inglês o termo "avaliação" (assessment) se refere apenas a avaliações prospectivas, a definição e metodologia trazida pelo Consenso faz todo sentido. O que se defende aqui, talvez pelo fato do termo “avaliação" ser, em português, mais abrangente, é que, ao apreender esse constructo sob um domínio mais amplo de abordagem, outras metodologias e definições podem ser entendidas como "avaliação de impacto à saúde".

Tal foi o impacto desse consenso que até hoje a definição mais difundida de AIS, bem como a metodologia mais utilizada, é aquela nele descrita. São vários os benefícios que a padronização pode trazer. Contudo, ela tende a diminuir a diversificação da prática e dificultar a expressão de outras formas de pensar e fazer. Essas questões reaquecem o debate sobre a delimitação da AIS e quais podem ser seus instrumentos.

Foram tais questões que motivaram a elaboração deste artigo, que busca resgatar a definição da AIS como um campo de saber, fundamentando seu amplo espectro de metodologias e práticas. Também busca-se expor práticas que compartilham o objetivo de promover saúde e reduzir os danos à qualidade de vida provocados por uma estratégia que poderia integrar o domínio mais amplo da avaliação de impactos à saúde.

\section{Avaliação de impacto à saúde como campo de saber}

O conceito de campo de saber presente na Lei de Diretrizes e Bases da Educação Nacional (LDB) do Brasil (Brasil, 1996) pode ser entendido, conforme parecer do Conselho Nacional de Educação de 1998 como "um recorte específico de uma área do conhecimento, ou de suas aplicações, ou de uma área técnico profissional ou, ainda, uma articulação de elementos de uma ou mais destas" (Brasil, 1998, p. 8). Com base nesse conceito, pode-se dizer que as origens da AIS, já relatadas na literatura (Harris-Roxas et al., 2012), servem como evidências que sustentam a argumentação desta como campo de estudo.

Ressalta-se que a caracterização da AIS como campo de saber não busca detê-la ao universo científico. O compartilhamento de conhecimentos no âmbito da AIS ocorre entre diferentes partes (Saarela; Söderman; Lyytimäki, 2015) e a geração de novos conhecimentos e construção de aprendizagens não se reduz ao universo acadêmico-científico (Sánchez; Mitchell, 2017). Scott-Samuel et al. (2001) chegam a afirmar que AIS não é estritamente uma ciência. Contudo, acredita-se ser possível que um campo do saber encontre suas fronteiras e significações além dos muros da academia.

De todo modo, é inegável que haja um interesse científico no domínio. Nas palavras de Kemm (2005), a AIS, como disciplina, está em bom estado de implementação, inclusive com boas perspectivas para o futuro. Exemplo disso são os artigos científicos sobre o tema que vêm sendo publicados ao longo do tempo por membros da academia. Outros exemplos são as disciplinas voltadas ao público acadêmico - como a oferecida no Swiss Federal Institute of Technology $(E T H)^{1}$-, os cursos de formação e divulgação da AIS como os desenvolvidos pela Organização Mundial de Saúde para funcionários de diversos ministérios oriundos de diferentes regiões, inclusive da América Latina (WHO, 200o) e o curso promovido pela Fundação Oswaldo Cruz² no Brasil, voltado também para a área técnica.

Buscou-se representar na Figura 1 a caracterização da AIS como campo de saber por meio de principais bases e pilares, organizando o referencial que aparece fragmentado em diversas publicações.

1 SWISS FEDERAL INSTITUTE OF TECHNOLOGY. Health impact assessment: concepts and case studies, 2016. Disponível em: <https:// goo.gl/J6yx3C>. Acesso em: 10 out. 2016.

2 FUNDAÇÃO OSWALDO CRUZ. Avaliação de impacto à saúde em países em desenvolvimento, 2015. Disponivel em: $\langle\mathrm{https://goo.gl/m3Zr5C}\rangle$. Acesso em: 10 jul. 2016. 


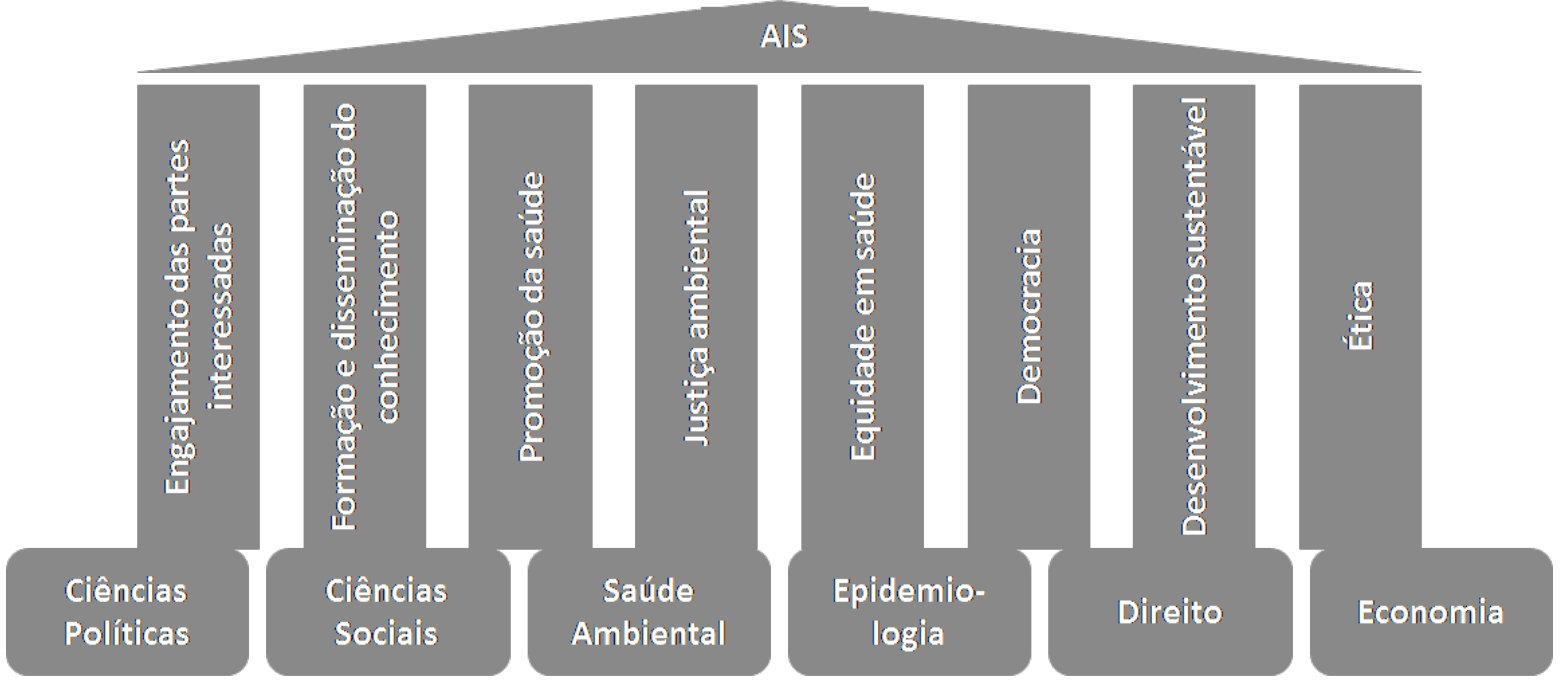

Fontes: Baseado em WHO, 1999; Pope et al., 2013; Harris-Roxas et al., 2012; Tamburrini; Gilhuly; Harris-Roxas, 20I1; Hurley; Vohra, 2010; Sánchez; Mitchell, 2016; Sicilia; Purroy, 2008.

Quanto aos pilares da AIS, que representam seus princípios de valor e preceitos, Tamburrini, Gilhuly e Harris-Roxas (2011) citam o engajamento das partes interessadas, o que deve incluir a participação comunitária, citada por Hurley e Vohra (2010). Sánchez e Mitchell (2016) destacam o papel da AIS na formação e disseminação do conhecimento; Sicilia e Purroy (2008) falam do preceito da promoção da saúde, e Hurley e Vohra (2010) citam justiça ambiental e equidade em saúde, o que inclui a abordagem dos determinantes sociais citados também por Harris-Roxas et al. (2012). No próprio consenso de Gothenburg (WHO, 1999) são citados como princípios da AIS a democracia, a equidade, o desenvolvimento sustentável e o uso ético das evidências. Esses princípios descrevem bem o propósito da AIS, em resposta à forma com que os desfechos de saúde decorrentes da exploração de recursos e da modificação do território vinham sendo tratados. Além disso, esses preceitos resgatam a essência dos movimentos de profissionais da saúde pública que muito contribuiu, segundo Harris-Roxas et al. (2012), para o desenvolvimento da AIS.

Já com relação às bases, Pope et al. (2013) descrevem que a teoria da avaliação de impactos evoluiu de campos já bem teorizados, dentre os quais as ciências políticas. Hurley e Vohra (2010), por sua vez, citam a saúde ambiental, a sociologia e a epidemiologia como ciências nas quais se encontram as raízes da AIS. Poder-se-ia adicionar as ciências do direito e da economia, uma vez que a primeira oferece as regras que nortearão a conduta (Nader, 2011), sobre as quais pensamentos sobre impactos e gestão serão elaborados; e a segunda, voltada a solucionar os problemas da luta pela formação de riquezas (Rossetti, 1977), oferece as bases para discussão sobre desenvolvimento, oportunidades e utilidades que se deseja ter, ou que se abre mão, ao decidir sobre a viabilidade de uma determinada estratégia.

Muitas outras áreas podem ser citadas ao se tratar das bases da AIS como campo de saber, ao passo que se lança mão de diversos conhecimentos e saberes para construir o pensamento sobre a interação entre as estratégias, o ambiente e a saúde humana. Por exemplo, antropologia, toxicologia, filosofia, biologia, geografia e história. Todas essas áreas oferecem conhecimentos que são utilizados pela avaliação de impactos à saúde em algum momento. Além disso, teorias como a da tomada de decisão e do planejamento são referenciadas como bases da avaliação de impacto (Leknes, 2001; Hildén; Furman; Kaljonen, 2004; Pope et al., 2013). Portanto, a representação contida na Figura 1 busca apenas ilustrar as principais bases e pilares da AIS, não se tratando de uma lista exaustiva, e sim exemplificativa e sumária. 
Na seção a seguir, buscou-se caracterizar a AIS como processo de estudo, entendendo que essa prática não se restringe ao âmbito acadêmico-científico e ultrapassa as teorias e metodologias sugeridas no Consenso de Gothenburg.

\section{A prática da avaliação de impacto à saúde como processo de estudo}

AAIS como processo de estudo tem sido realizada em diferentes países e em variadas circunstâncias. Erlanger et al. (2008) demonstraram diferentes aplicações dessa avaliação ao redor do mundo, enquanto Pereira et al. (2017) demonstraram a aplicação da AIS na América Latina. Com base em um entendimento ampliado da AIS como processo sistemático de análise materializado em estudos, pode-se dizer que este se volta a orientar a prevenção, a gestão e compensação dos danos à saúde (impactos negativos), e a promoção dos impactos positivos, subsidiando a tomada de decisão em qualquer fase do ciclo de vida de uma estratégia. A AIS tem sido utilizada principalmente em duas grandes vertentes: aplicada à avaliação de políticas públicas e de projetos econômicos ou de desenvolvimento (Rajotte et al., 2011).

A proposição de Campos et al. (1997) em diferenciar campo e núcleo pode ser útil para organizar o pensamento em torno do que se defende aqui. Segundo os mesmos autores, campo seria um espaço de limites e contornos menos precisos em que ocorre a intersecção com outras áreas. Dentro do campo estão os núcleos, cujos limites são bem definidos e que dão forma a uma especialidade e demarcam uma identidade. Neste caso, tomando-se o constructo mais amplo da AIS como campo e a dimensão trazida pelo consenso de Gothenburg como núcleo de práticas em AIS, restaria identificar quais outros núcleos poderiam ser reconhecidos. Neste sentido, a Figura 2 representa o campo da AIS e os núcleos de práticas identificados como parte do arcabouço aqui defendido.

Sob essa égide, estudos vinculados às avaliações de impacto ambiental, seguindo ou não os passos definidos pelo Consenso de Gothenburg, trabalhos de monitoramento não vinculados a estudos prospectivos anteriores, avaliações de risco e avaliações econômicas poderiam ser entendidos como estudos de avaliação de impactos sobre a saúde. Todas essas abordagens podem ser evocadas para abordar qualitativa e/ou quantitativamente as questões relativas à saúde humana que decorrem da implementação de estratégias e da consequente modificação do ambiente e do território. Até mesmo os estudos situacionais e os de vulnerabilidade poderiam ser entendidos como parte do processo de análise de impactos. Assim, haveria uma variedade de formas de se materializar em estudos o pensamento sobre impactos contido no domínio do campo da AIS.

\section{Figura 2 - Caracterização do campo da avaliação de impacto à saúde e dos núcleos ligados a esse campo}

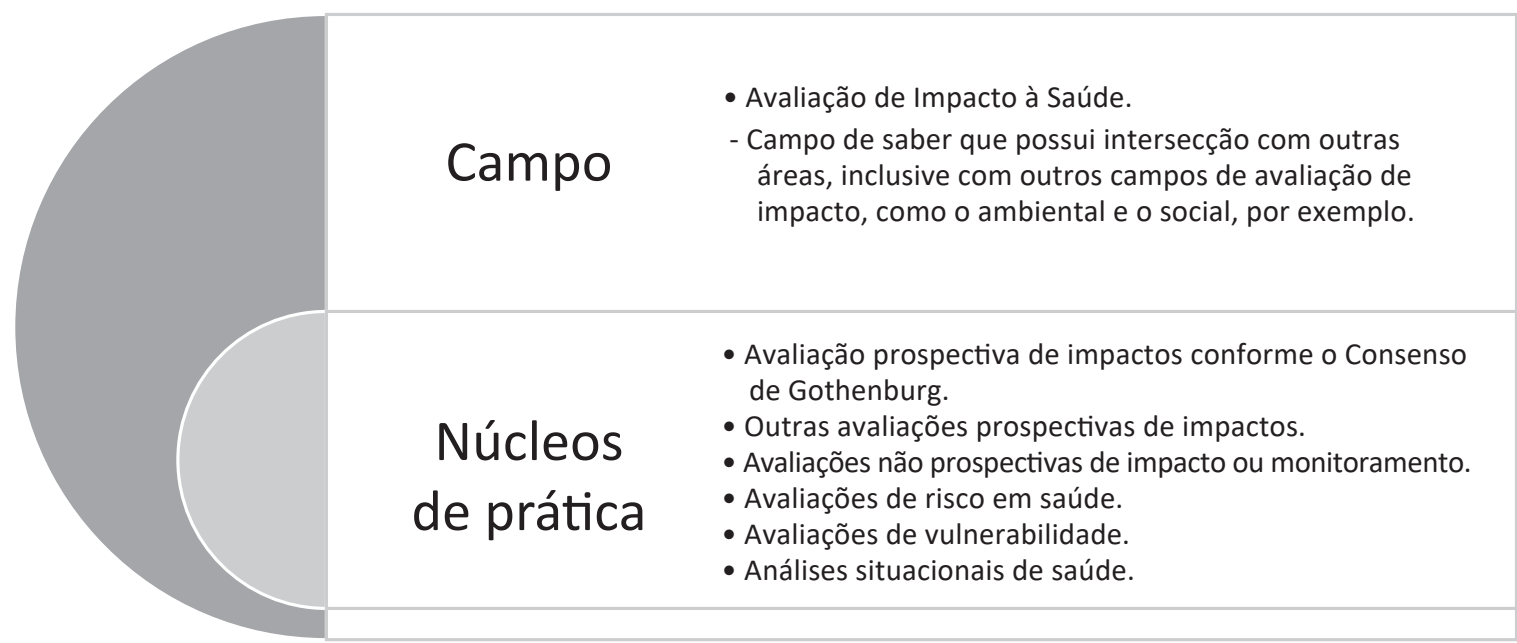

Fonte: Baseado nas definições de campo e núcleo trazidas por Campos, 1997. 
O campo aqui defendido foi chamado de Avaliação de Impacto à Saúde (AIS). 0 termo "avaliação" tanto no português, como no espanhol e no francês, refere-se ao processo de análise, seja prospectivo ou não. Diferentemente, no inglês, existe distinção de termos utilizados para designar avaliações prospectivas (assessments) e as não prospectivas (evaluations). A tradução deste texto para o inglês exigiria uma adaptação do termo utilizado para definir o campo, uma vez que avaliação (assessment) não serviria para representar o universo do campo aqui defendido. Talvez, seria o caso de nomear o campo como health impact appraisal, ou health impact examination.

\section{Considerações finais}

No decorrer deste estudo, fundamentou-se a AIS como campo de saber, considerando que existe um sistema composto por um aparato teórico - conhecimentos, princípios, preceitos, funções e regras que servem para lhe dar corpo e delimitação - e uma estrutura formada por instituições e atores em atividade. Ademais, argumentou-se que a prática de avaliação de impactos à saúde pode ocorrer via outras metodologias que não a difundida pelo Consenso de Gothenburg.

Buscou-se fomentar a discussão sobre algo latente, lacunas que permeiam a prática da análise de impactos à saúde, e que, de certa forma, interfere no modo como o pensamento sobre impactos é estruturado e como são superadas as limitações de método. Dessa forma, espera-se que a reflexão sobre aspectos teóricos e práticos que permeiam o construto da AIS possam contribuir para a expansão do campo e o amadurecimento da prática.

\section{Referências}

BRASIL. Lei no ${ }^{\circ}$ 9.394, de 20 de dezembro de 1996. Estabelece as diretrizes e bases da educação nacional. Diário Oficial da União, Brasília, DF, 23 dez. 1996. Seção 1, p. 27833.

BRASIL. Ministério da Educação e do Desporto. Conselho Nacional de Educação. Parecer homologado. Retificação do Parecer CES $n^{0} 672 / 1998$, tratando de Cursos Sequenciais no
Ensino Superior. Diário Oficial da União, Brasília, DF, 23 dez. 1998.

CAMPOS, G. W. S.; CHAKOUR, M.; SANTOS, R. C. Análise crítica sobre especialidades médicas e estratégias para integrá-las ao Sistema Único de Saúde (SUS). Cadernos de Saúde Pública, Rio de Janeiro, v. 13, n. 1, p. 141-144, 1997. Disponível em: <https://goo.gl/dLGssB>. Acesso em: 15 set. 2016.

DONELLY, A.; DALAL-CLAYTON, B.; HUGHES, R. (Ed.). A directory of impact assessment guidelines. 2. ed. Nottingham: Russell Press, 1998.

ERLANGER, T. E. et al. The 6/94 gap in health impact assessment. Environmental Impact Assessment Review, Amsterdam, v. 28, n. 4-5, p. 349-358, 2008.

HARRIS-ROXAS, B. et al. Health impact assessment: the state of the art. Impact Assessment and Project Appraisal, Abingdon, v. 30, n. 1, p. 43-52, 2012.

HILDÉN, M.; FURMAN, E.; KALJONEN, M. Views on planning and expectations of SEA: the case of transport planning. Environmental Impact Assessment Review, Amsterdam, v. 24, n. 5, p. 519536, 2004 .

HURLEY, F.; VOHRA, S. Health impact assessment. In: AYRES, J. G. et al. Environmental medicine. Boca Raton: CRC Press, 2010. p. 666-678.

KEMM, J. HIA: growth and prospects. Environmental Impact Assessment Review, Amsterdam, v. 25, n. 7-8, p. 691-692, 2005.

LEKNES, E. The roles of EIA in the decisionmaking process. Environmental Impact Assessment Review, Amsterdam, v. 21, n. 4, p. 309-334, 2001.

NADER, P. Filosofia do direito. 20. ed. Rio de Janeiro: Forense, 2011.

POPE, J. et al. Advancing the theory and practice of impact assessment: setting the research agenda. Environmental Impact Assessment Review, Amsterdam, v. 41, p. 1-9, 2013.

PEREIRA, C. A. R. et al. Health impact assessment in Latin American countries: current practice and 
prospects. Environmental Impact Assessment

Review, Amsterdam, v. 65, p. 175-185, 2017.

RAJOTTE, B. R. et al. Health in all policies: addressing the legal and policy foundations of health impact assessment. The Journal of Law, Medicine \& Ethics, Medford, v. 39, n. 1, p. 27-29, 2011. Supplement 1.

ROSSETTI, J. P. Introdução à economia. 6. ed. São Paulo: Atlas, 1977.

SAARELA, S.-R.; SÖDERMAN, T.; LYYTIMÄKI, J. Knowledge brokerage context factors - What matters in knowledge exchange in impact assessment? Environmental Science \& Policy, Amsterdam, v. 51, p. 325-337, 2015. Disponível em: <https://goo.gl/x4mLAA>. Acesso em: 10 set. 2016.

SÁNCHEZ, L. E.; MITCHELL, R. Conceptualizing impact assessment as a learning process. Environmental Impact Assessment Review, Amsterdam, v. 62, p. 195-204, 2017. Disponível em: <https://goo.gl/ksisto>. Acesso em: 23 set 2016.
SCOTT-SAMUEL, A.; BIRLEY, M.; ARDERN, K. The merseyside guidelines for health impact assessment. 2. ed. Liverpool: International Health Impact Assessment Consortium, 2001.

SICILIA, A. R.; PURROY, C. A. La evaluación del impacto en salud: el estado de la cuestión. Gaceta Sanitaria, Barcelona, v. 22, n. 4, p. 348-53, 2008.

TAMBURRINI, A.-L.; GILHULY, K.; HARRISROXAS, B. Enhancing benefits in health impact assessment through stakeholder consultation. Impact Assessment and Project Appraisal, Abingdon, v. 29, n. 3, p. 195-204, 2011.

WHO - WORLD HEALTH ORGANIZATION. Who Regional Office for Europe. Health Impact Assessment: main concepts and suggested approach. Gothenburg consensus paper. Brussels: WHO, 1999. 10p.

WHO - WORLD HEALTH ORGANIZATION. Intersectoral decision-making skills in support of health impact assessment of development projects. Genève/Charlottenlund: WHO, 2000.

\section{Contribuição dos autores}

Pereira foi responsável pela concepção do estudo. Hacon selecionou referências para elaboração do texto. Ambos os autores contribuíram para a redação do artigo.

Recebido: 14/02/2017

Reapresentado: 25/08/2017

Aprovado: 28/08/2017 